\title{
Peer to Peer Proxy based IPTV Services
}

\author{
Shiddhartha Raj Bhandari, Gyu Myoung Lee, and Noel Crespi \\ Department of Wireless Networks and Multimedia Services \\ Institute TELECOM, TELECOM SudParis \\ 9 rue Charles Fourier, 91011, Evry, France \\ \{Shiddhartha_raj.Bhandari,gm.lee, noel.crespi\}@it-sudparis.eu
}

\begin{abstract}
As Internet Protocol Television (IPTV) is becoming popular as a medium of seamless infotainment, service providers are being attracted in IPTV as they can aggregate different application services on top of it and users are interested in interactive services using video on demand (VoD), trick mode functionalities on live TV etc. Service delivery latency is an important issue in providing interactive live TV broadcasting. In this paper, we aim to reduce channel zapping time and channel play back period using benefits of Peer to peer (P2P) and proxy server. For this, we propose a new P2P proxy based IPTV service delivery architecture which provides TV channel and VoD service with minimum delay. P2P enabled proxy server reduces signaling overhead and provides quick service delivery. We perform delay comparison of our proposed solution with currently available system in order to show considerable delay gain of our architecture.
\end{abstract}

Keywords-IPTV; Content; Peer-to-Peer; Proxy Server

\section{INTRODUCTION}

Increasing popularity of multimedia communication has opened various opportunities. Video content distributions, TV channel distribution, online gaming are few examples. Along with new opportunities, it also posses challenges in efficient content distribution. These applications are bandwidth consuming and can not tolerate the high delay and poor loss property of today's IP network [1].

Internet Protocol Television (IPTV) has opened new avenue for both content providers and network providers [2]. Content providers get benefitted by selling their content while network providers get benefitted using their network as means of carrying content form content providers to end users. These days more and more users are being interested in digital TV services than traditional cable or satellite broadcasting. Voice, video and data communication services are reaching home through a single broadband communication. One important reason that lured users in this service is better quality of video and sound in digital form.

Interactive IPTV which allows users to perform trick mode operations (e.g., play, pause, rewind, time set, etc) is another hot research and implementation area. Many service providers have already started providing interactive IPTV services to their end users. Users can perform trick mode operation like pause, rewind when watching live channel. When someone missed some TV show, he or she just can press remote control rewind TV channel to desired time and watch in their feasible time.

Although interactive IPTV started, it has many challenges to solve. Corporate and academic researchers are working on it to make it really feasible for all TV viewers. In this research, we are focusing on some of the relevant problems related to trick mode and time shifted IPTV service delivery. Service delivery latency is the important problem that should be given high priority. When user is watching a channel and changes to another, it should not take long time to start. Although there are many signaling and network related operations to do, it should be done quickly with some efficient techniques. Therefore, the problem related to maintaining the quality of experience (QoE) is still there and need to explore more.

Current IPTV architecture has considerable service delivery latency. Channel zapping time, which is the time duration taken by IPTV system to switch from one channel to another, is quiet high. Video and TV contents are sensitive to delay, which degrades QoE if it is higher than certain threshold. In this paper, we propose a novel proxy based IPTV delivery architecture. Proxy servers are distributed strategically to cover service deployment areas. Peer-to-Peer (P2P) enabled proxies provide lower latency service delivery to requesting customer. The proposed architecture reduces control signaling overhead compare to other delivery architectures. Use of high speed prefix caching provides quick channel delivery while supporting trick mode operations. Each proxy server has its own storage unit to store contents when users start trick mode operations. Peer proxies co-operate each other in content delivery without redundancy of channel storage. Requested channel will be retrieved form peer proxy first and then from head end server if it is not available in its peer. The aim of our paper is to achieve performance improvement in terms of delay in content delivery for IPTV services considering user's dynamic behaviors and a number of TV channels.

The remainder of this paper is organized as follows. Section II introduces content delivery architectures proposed for IPTV services. Section III describes proposed P2P proxy based IPTV delivery architecture. Content delivery mechanism of proposed architecture is discussed in Section IV. Comparison of proposed system is done in Section V. We conclude paper in Section VI discussing some challenges and our future research steps. 


\section{RELATED WORK}

\section{A. Content Delivery Architecture for IPTV Services}

1) Server based architecture(Figure 1. (a))

Server based IPTV service delivery architecture is similar to an architecture developed to provide video on demand (VoD) service over internet. It consists of content distribution server at the head end and service requesting clients with settop-box (STB) in the edge of the network.

2) Content distribution network architecture (Figure 1.(b))

Content distribution network (CDN) is a kind of network consisting of distributed content distribution servers. CDN architecture consists of main content sever, and strategically placed content distribution servers called surrogates. When user requests content, system redirects that request to one of the surrogates which is near to the user. Metrics used in deciding request redirection can be hope count from client to surrogate or delay that may occur during transmission. CDN architecture is a potential candidate in providing interactive IPTV service delivery. CDN surrogates which are distributed globally can provide low latency digital TV service to requesting customer.

3) Peer-to-peer based architecture (Figure 1. (c))

The great success in file sharing application has moved P2P system toward real time video streaming services [3]. P2P technology has greatly enhanced the distribution of information enabling efficient co-operation among end users. As there is increment in network bandwidth because of broad band connections, P2P is becoming a scalable solution to provide interactive IPTV and VoD services. P2P networks have shown to be a promising approach to provide large-scale VoD services over the internet as it can handle the main problem of centralized delivery system, load balancing and scalability. This paper selected P2P based architecture as it provides scalable solution for interactive IPTV service delivery.

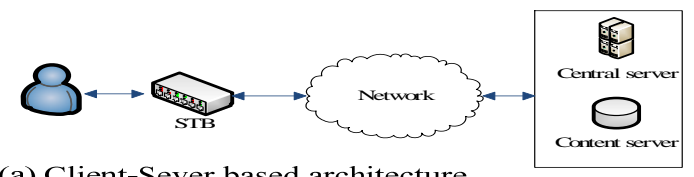

(a) Client-Sever based architecture

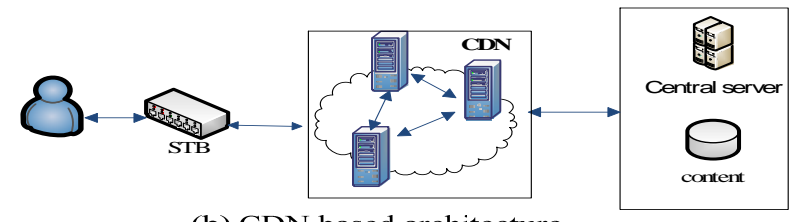

(b) CDN based architecture

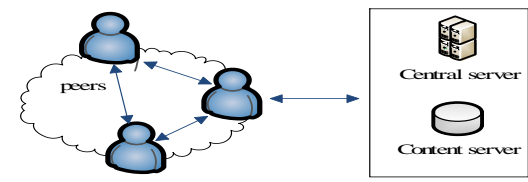

(c) Peer-to-Peer based architecture

Figure 1. Content delivery architectures for IPTV services

\section{B. Content delivery mechanisms using proxy server}

Usefulness of proxy server has already been observed in case of World Wide Web and VoD service delivery [4]. Proxy servers are deployed between the main server and clients so that clients request can be served without contacting specified server. Proxy caching has already been proposed for IP multimedia streaming [5][9]. Proxy stores recently accessed resources and serves future client requests without contacting the server.

Multimedia processing proxy has been proposed for IPTV service delivery in ambient network [6]. Proxy server adapts content received from the server so that all kinds of devices can access IPTV services seamlessly. Fast mesh project [7] has also started developing P2P overlay proxy based IPTV service delivery. User terminal and proxy server of IPTV system has already been patented in Korea [8].

Many researchers have tried to reduce large play back delay with various mechanisms. Segmenting, piggybacking, patching, stream tapping are some examples. One of them is proxy based prefix caching [9]. In this mechanism, proxy server resides in between client and main multimedia server and caches prefix of video content so that it can responds to video request without causing delay. This paper has considered a prefix caching mechanism in order to get delay gain.

\section{PeER to PeER Proxy BAsed IPTV ARChitecture}

\section{A. Peer-to-Peer Proxy Model}

Proxy based approaches were initially proposed and found to be useful in delivering web objects [10]. It reduces load in centralized server providing requested web components itself rather than routing it all the way to the main server. When VoD service started on top of internet, the same proxy based approach has been adopted. Proxy servers are deployed between the server and remote clients. By using proxy based approach, service providers get benefit of accelerated video playback and reduction in network bandwidth.

We believe that there should be some intermediate content distribution entities between main content server and end user to provide live IPTV services. P2P based proxy servers lie within the closed network environment of service provider and relay live TV channels to end user quickly. Each peer will have its own capacity to handle requesting users. Intermediate proxy servers are deployed which keep available all the channels being offered in advance. When user requests new channel or perform interactive operations, proxy server handles all control and media delivery functions. It reduces service delivery latency with considerable level. It also reduces the probability of requests or media stream packets getting lost with in large scaled IP network.

Figure 2 shows P2P proxy based IPTV architecture. Proxy servers form middle level service delivery platform. Live TV channels and VOD contents are being served from video head end (VHO) using multicast channel. Users are associated with their own proxy servers and do not know the upper layer abstraction, they just need to request channel using remote control. Proxy servers are connected with each other using P2P network. Each proxy might be serving different channels depending on viewing behavior of currently serving users. 


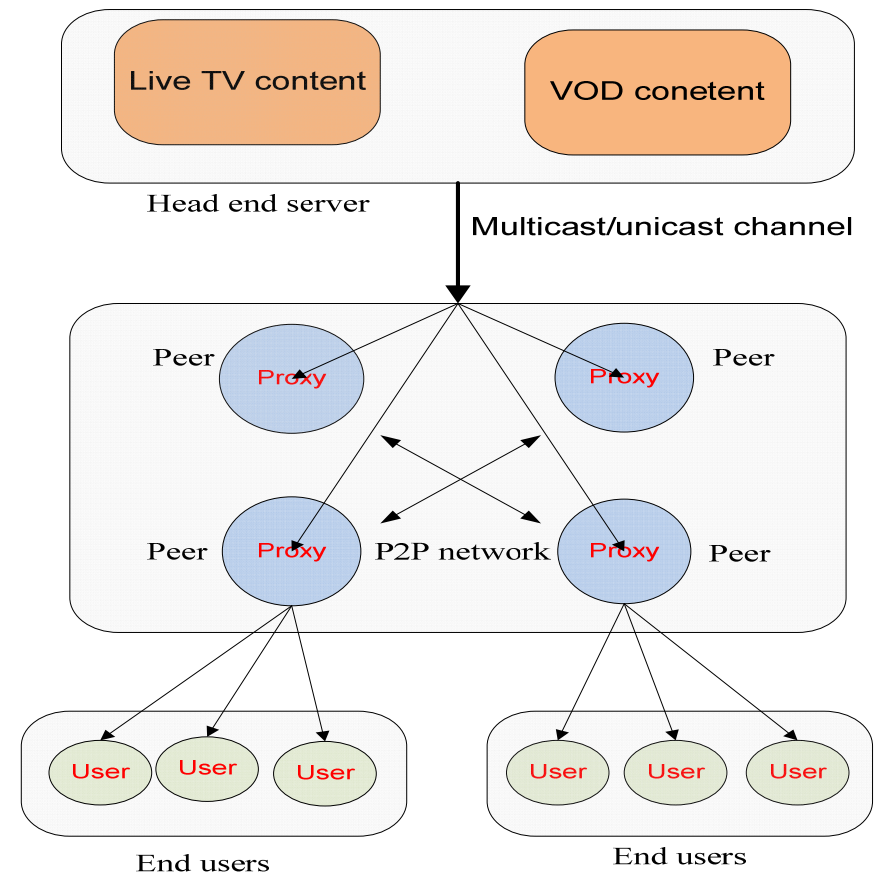

Figure 2. Peer-to-peer proxy based IPTV architecture

\section{B. Logical Channel Seperation for Content Delivery}

IPTV service delivery requires supporting diverse client server interactions and should provide some stringent demands. Protocol suite that enables such facilities in control and media plane is required. Internet Engineering Task Force (IETF) has developed protocol suite to be used for content delivery. It consists of data and control channel.

- Data channel for content delivery

Data transfer is done using real time protocol (RTP). RTP provides payload identification, sequence numbering and time stamping which help in playback controlling.

- Control channel for content delivery

Control channel is consists of real time control protocol (RTCP) and Real time streaming protocol (RTSP). RTCP helps monitoring the network status and provides feedback control during transmission. RRTSP provides set of controls for interactive play back.

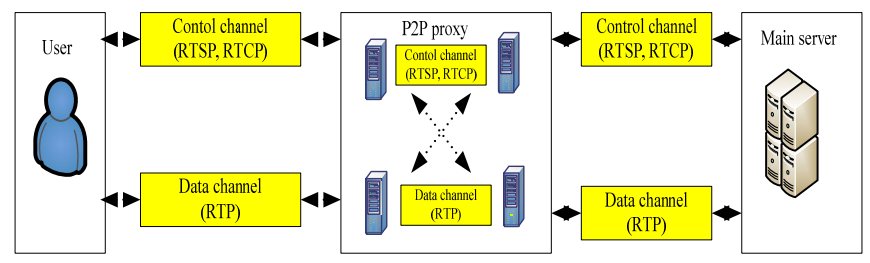

Figure 3. Control and data channel for content delivery

\section{Fuctional Architecture for Peer-to-Peer based Proxy}

This section describes different functional components of P2P based proxy server. Proxy servers form content delivery platform with P2P networking among each others. Each proxy server is capable of handling user generated interactive IPTV requests with minimum service delivery latency. Proxy server consists of various sub constituents which perform their own task. Authentication unit is responsible for new or current user authentication and channel management unit maintains information about current and newly requested channel availability. Cache and storage management unit manages high speed content caching and storage of TV program if it is required. User behavior monitoring unit monitors viewing statistics and channel changing behavior of users. Lookup unit helps finding which peer has requested channel information and Interface unit retrieves channel which are present in peer proxy or VHO. Figure 4 shows different functional components of P2P enable proxy server. Table I describes them briefly.

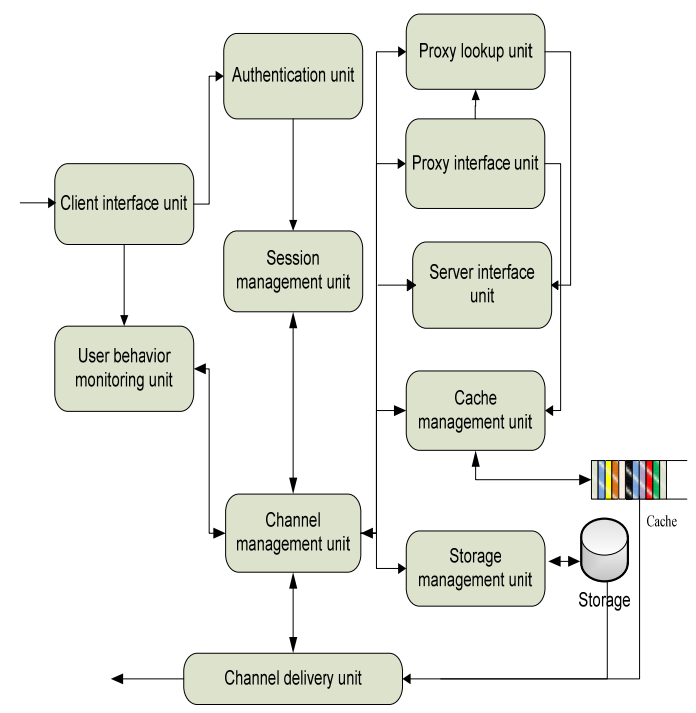

Figure 4. Functional architecture of P2P based proxy

TABLE I. FUCNTIONAL DESCRIPTION OF P2P BASED PROXY SERVER

\begin{tabular}{|l|l|}
\hline \multicolumn{1}{|c|}{ Component } & \multicolumn{1}{c|}{ Description } \\
\hline User interface unit & Process user's IPTV requests \\
\hline Authentication unit & Authenticate requesting user \\
\hline $\begin{array}{l}\text { Session management } \\
\text { unit }\end{array}$ & $\begin{array}{l}\text { Perfom session setup signaling with peer proxy } \\
\text { head end server and requesting user }\end{array}$ \\
\hline $\begin{array}{l}\text { User behavior } \\
\text { monitoring unit }\end{array}$ & $\begin{array}{l}\text { Monitor user's preferences and channel } \\
\text { viewining behavior }\end{array}$ \\
\hline $\begin{array}{l}\text { Channel management } \\
\text { unit }\end{array}$ & $\begin{array}{l}\text { Keep information about currently serving and } \\
\text { user requested channels }\end{array}$ \\
\hline Proxy lookup unit & $\begin{array}{l}\text { Perform lookup to find the peer proxy which has } \\
\text { requested channel }\end{array}$ \\
\hline Server interface unit & $\begin{array}{l}\text { Request head end serverif requested channel is } \\
\text { not available in peer proxy }\end{array}$ \\
\hline Cache mangement unit & $\begin{array}{l}\text { Manage caching of currently delivered channel. } \\
\text { Cache new channel from peer proxy or VHO }\end{array}$ \\
\hline $\begin{array}{l}\text { Storage management } \\
\text { unit }\end{array}$ & $\begin{array}{l}\text { Store content when interactive requests take } \\
\text { place (pause, stop) }\end{array}$ \\
\hline Channel delivery unit & Deliver available channel to authentiacted users \\
\hline
\end{tabular}




\section{Contnet Delivery Mechanism using Peer to Peer PROXY}

In the proposed architecture, we are describing detail operation according to different use cases. There are three possible cases;

- Case I: user requested service is available in proxy server

- Case II: user requested service is available in peer proxy

- Case III: service is required to be served from main head end server

Figure 5 shows operations of Case I, proxy server has user requested channel. When user generates service request through remote control, proxy server authenticates requesting user and sends immediately the requested TV channel through its cache memory. In this case there is no signal overhead from one proxy to another and head end server.

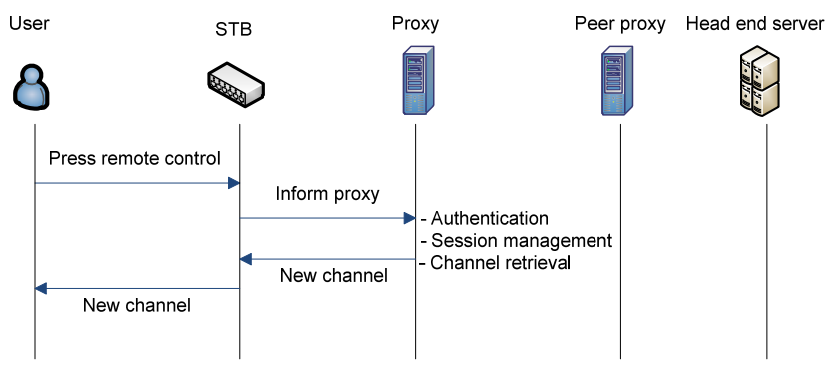

Figure 5. Channel is available in proxy server (Case I)

As shown in Figure 6, Case II describes when a requesting proxy does not have requested TV channel. In this case, proxy server requests another peer for requested content. Lookup unit of proxy server quickly finds peer that has requested channel content. Compared with Case I, signaling and caching overhead will be added in proxy server.

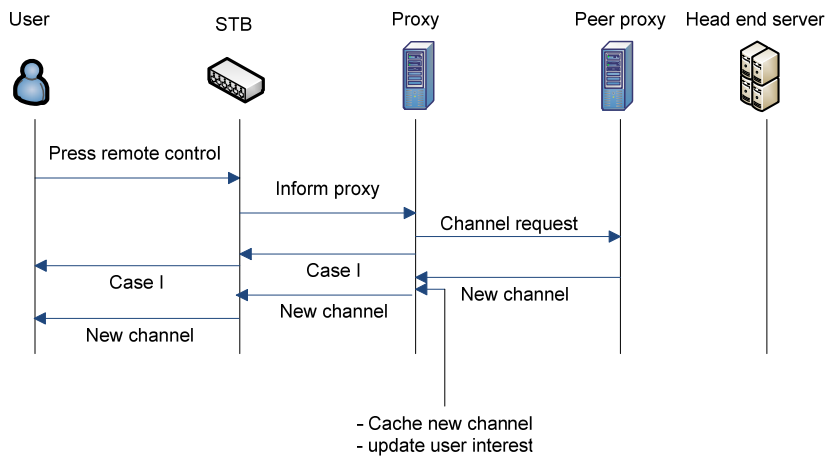

Figure 6. Channel content retrieves from peer proxy (Case II)

As shown in Figure 7, Case III shows the operations of the case in which content isn't available and need to retrieve from head end server. In this case, signaling is required between proxy and head end server. Compared with Case I and Case II, signaling and media retrieval delay between proxy and head end server is added for this case.

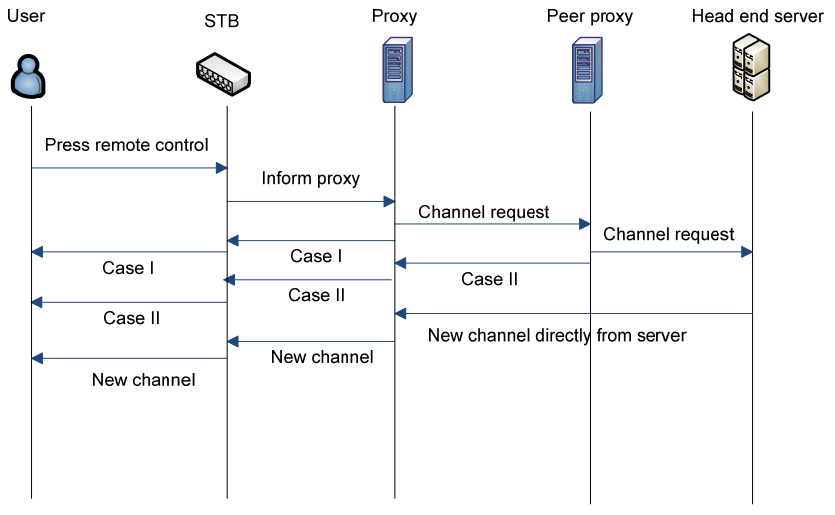

Figure 7. Channel retrieves from head end server (Case III)

Figure 8 shows detail operations of IPTV service delivery.

Step 1: user starts interactive operation through its remote control

Step2: STB routes the request to proxy server

Step 3: Authentication unit performs user

authentication to find whether that user is

authenticated for that operation

Step 4: Channel management unit checks

availability of requested channel

Case I: Channel is present in proxy server

Step 5: Cache management unit perform caching

look up for that channel

Step 6: Channel delivery unit immediately start

sending video frames to the customer STB

Step 7: Session management unit performs

establishing new session, inform user behavior

monitoring unit about new session for that

particular user

Step 8: when session management and signaling

operation finished user will be served through

live channels rather than cache memory

Case II: channel is present in Peer-proxy

Step 5: Proxy lookup unit performs which proxy

server has requested content

Step 6: Requested content is received by proxy

interface unit and inform it to cache management,

channel management, and channel delivery unit

Step 7: Channel delivery unit starts sending

content, cache management unit starts putting

content in cache, session management unit start

managing new session, user behavior monitoring

unit keeps this record

Case III: Channel is present in main content feed server

Step 5: Server interface unit requests content of requested channel

Step 6: Server interface unit gets channel

content from content feed server. Inform cache

management, channel management, and channel

delivery unit

Step 7: Channel delivery unit start sending

content, cache management unit start putting

content in cache, session management unit start

managing new session, user behavior monitoring

unit keeps this record

Figure 8. Deatialed operations for P2P proxy based IPTV services 
Figure 9 shows the detail flow chart of P2P proxy based IPTV service delivery architecture with the alignment of proposed functional components of P2P based proxy system.

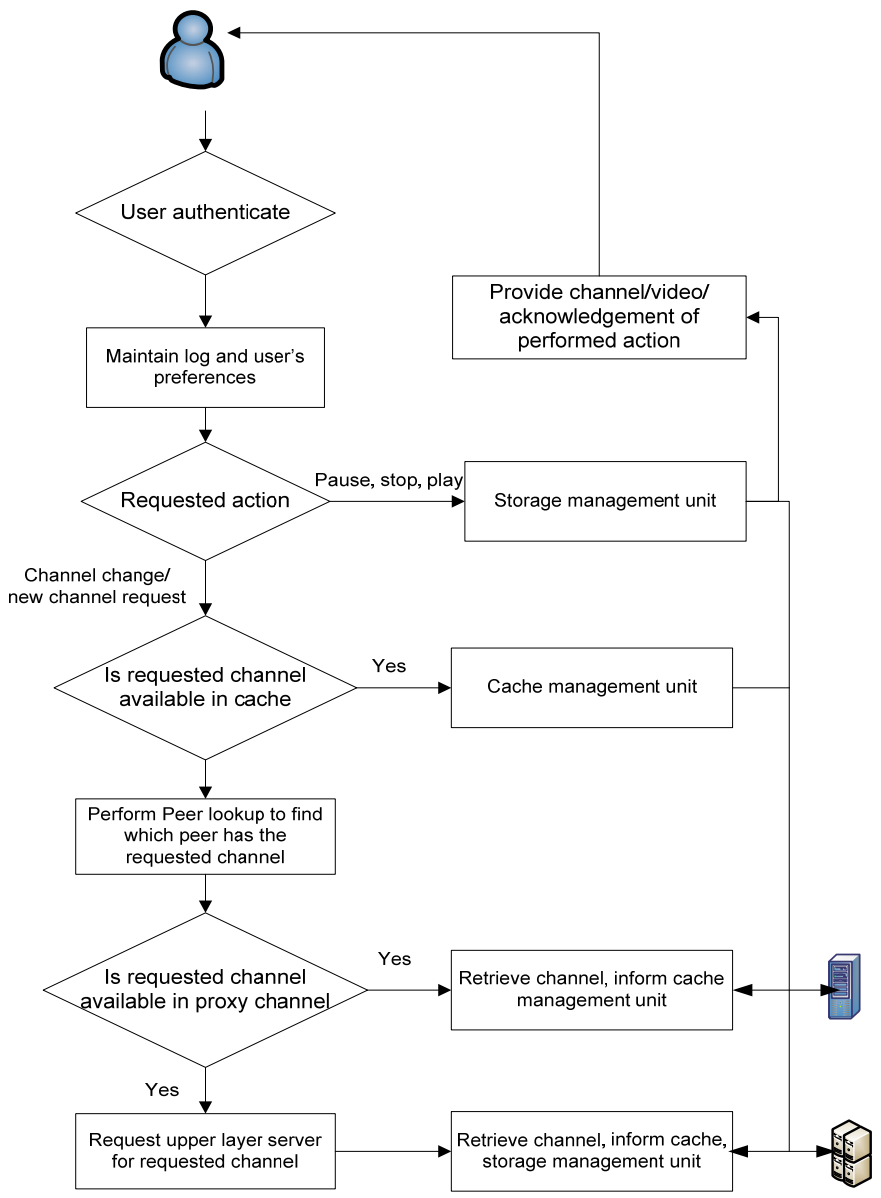

Figure 9. Flow chart for P2P proxy based IPTV services

\section{Performnace COMPARISON}

\section{A. Delay Characteristics for IPTV Content Delivery}

Channel zapping delay is a key of experience metric in IPTV [11]. It is defined as the difference between the user presses remote control to request a new channel and starts displaying it in TV screen. IPTV delivery architecture consists of various components at various levels. Each component requires signaling and takes few milliseconds to finish its task. Components that cause delay in content delivery for IPTV services are as follows;

- $\quad$ Signaling delays

When user performs some operations on TV channel, it requires leaving current multicast group and joining a new. Few milliseconds spend in this process.

- Administrative and content related delay

This delay is related to contents that are being requested by user. Before serving channel, users may needed to be authenticated, some information may need to be processed which may take some more time causing delay.

- Network delay

IP network consists of various routers and switches. Channel change request may need to go through several hierarchical levels. IP packets may travel through different routes which might be congested or slow. In that case packets can stick in congestion or lost because of network failure. Therefore, it is very difficult to express exact network delay that might occur.

- $\quad$ Buffering delay

Buffering is required before starting to display received video frames. To play video smoothly, certain number of frames are required to be presented in buffer. It also takes considerable amount of time. We can not reduce buffering time but it also depends on the network bandwidth. If network bandwidth is high enough, number of frames required for buffering can reach destination quickly.

\section{B. Delay gain using prefix caching}

Proxy servers cache a fixed set of frames at the beginning of each popular video (i.e., prefix of the content), instead of storing the entire resource. In order to get delay gain, we propose to use of prefix caching on P2P based proxy architecture. Each proxy server stores certain duration of live video in their cache memory. When requests arrive, proxy server sends current live TV frames from cache memory immediately. Prefix caching reduces the problem of TV play back latency in current architectures.

\section{Delay Comparison}

We have finished discussion about various delay components that occur in IPTV service delivery. Now, we are comparing delay between our proposed proxy based architecture and delivery system that are currently being used. Delay varies depending on three different cases which specified in previous section.

Table II describes the case when user requests new or changes current channel and requested channel is available in proxy server. Current delivery system takes approximately 1330 milliseconds in normal condition neglecting some uncertain behavior of IP network [11]. Proxy based system excludes signaling and session setup delay using proxy server with prefix caching. Proxy server immediately starts to send TV content when authentication unit authenticates requesting users. It does not take long time for channel delivery unit to retrieve requested frames from buffer and send to customer end. Some signaling operations such as session setup, multicast join, merging, patching, and batching will be handled by session management unit in parallel with data transfer which reduces considerable amount of signaling delay. Peer proxies are deployed nearer to client access network, which reduces delay of delivering frames to user STB. 
TABLE II. CASE I: CHANNEL RETRIEVAL FROM PROXY CACHE

\begin{tabular}{|l|c|c|c|}
\hline \multicolumn{2}{|c|}{ Delay(ms) } & $\begin{array}{c}\text { Current } \\
\text { delivery system }\end{array}$ & $\begin{array}{c}\text { P2P Proxy } \\
\text { based system }\end{array}$ \\
\hline $\begin{array}{l}\text { Signaling delay } \\
\text { (remote control to } \\
\text { STB) }\end{array}$ & 10 & Yes & Yes \\
\hline $\begin{array}{l}\text { Signaling delay from } \\
\text { STB to proxy }\end{array}$ & 10 & Yes & Yes \\
\hline $\begin{array}{l}\text { Session management } \\
\text { delay (IGMP join, } \\
\text { stream tapping, } \\
\text { merging etc ) }\end{array}$ & 50 & Yes & No \\
\hline $\begin{array}{l}\text { Proxy buffer accessing } \\
\text { delay }\end{array}$ & minimum & No & Yes \\
\hline $\begin{array}{l}\text { Frame transmission } \\
\text { delay }\end{array}$ & 150 & $\begin{array}{c}\text { Yes } \\
\text { (variable })\end{array}$ & Fixed \\
\hline Buffering delay & 100 & Yes & Yes \\
\hline Decoding delay & 10 & Yes & Yes \\
\hline
\end{tabular}

Table III compares delay for the case when content is not available in requested proxy. In this case, proxy performs peer lookup and finds which has this content right now. It retrieves content from that peer and sends it to the requesting user. Delay occurs during peer lookup and data retrieval. However, this delay can be predicted and maintain at low level with high speed communication link.

TABLE III. CASE II: CHANNEL RETRIEVAL FROM PEER PROXY

\begin{tabular}{|l|c|c|c|}
\hline \multicolumn{2}{|c|}{ Delay(ms) } & $\begin{array}{c}\text { Current } \\
\text { delivery system }\end{array}$ & $\begin{array}{c}\text { P2P Proxy } \\
\text { based system }\end{array}$ \\
\hline $\begin{array}{l}\text { Signaling delay } \\
\text { (remote control to } \\
\text { STB) }\end{array}$ & 10 & Yes & Yes \\
\hline $\begin{array}{l}\text { Signaling delay from } \\
\text { STB to proxy }\end{array}$ & 10 & Yes & Yes \\
\hline $\begin{array}{l}\text { Session management } \\
\text { delay (IGMP join, } \\
\text { stream tapping, } \\
\text { merging etc ) }\end{array}$ & 50 & Yes & Nos \\
\hline $\begin{array}{l}\text { Proxy buffer accessing } \\
\text { delay }\end{array}$ & minimum & No & $\begin{array}{c}\text { Fixed } \\
\text { (minimum) }\end{array}$ \\
\hline $\begin{array}{l}\text { Frame transmission } \\
\text { delay }\end{array}$ & 150 & $\begin{array}{c}\text { Yes } \\
\text { (variable })\end{array}$ & Yes \\
\hline Buffering delay & 100 & Yes & Yes \\
\hline Decoding delay & 10 & Yes & \\
\hline
\end{tabular}

Table IV shows worst case delay that may occur when the requested channel is not present in peer proxies and needs to be retrieved from head end server. This takes more time compared with Case I and Case II. However, this delay is also predictable and can be maintained at certain level using high speed communication link between proxy servers and head end server.

TABLE IV. CASE III: CHANNEL RETRIEVAL FROM HEAD END SERVER

\begin{tabular}{|l|c|c|c|}
\hline \multicolumn{2}{|c|}{ Delay (ms) } & $\begin{array}{c}\text { Current } \\
\text { delivery system }\end{array}$ & $\begin{array}{c}\text { P2P Proxy } \\
\text { based system }\end{array}$ \\
\hline $\begin{array}{l}\text { Signaling delay ,remote } \\
\text { control to STB }\end{array}$ & 10 & Yes & Yes \\
\hline $\begin{array}{l}\text { Signaling delay from STB } \\
\text { to proxy }\end{array}$ & 10 & Yes & Yes \\
\hline $\begin{array}{l}\text { Session management delay } \\
\text { (IGMP join, stream } \\
\text { tapping, merging etc ) }\end{array}$ & 50 & Yes & $\begin{array}{c}\text { Yes } \\
\text { to peer } \\
\text { proxy) }\end{array}$ \\
\hline $\begin{array}{l}\text { Proxy buffer accessing } \\
\text { delay }\end{array}$ & minimum & No & Yes \\
\hline
\end{tabular}

\begin{tabular}{|l|c|c|c|}
\hline Frame transmission delay & 150 & Yes (variable ) & $\begin{array}{c}\text { Fixed } \\
\text { (minimum) }\end{array}$ \\
\hline Buffering delay & 100 & Yes & Yes \\
\hline Decoding delay & 10 & Yes & Yes \\
\hline
\end{tabular}

From our delay comparisons, we found that IPTV channel zapping time or service delivery latency can be reduced to some minimum level. Using P2P based proxy in between user and head end server, delay that occurs in case of current service delivery system is minimized.

\section{CONCLUSION}

In this paper, we have proposed P2P proxy based content delivery architecture for IPTV services which reduces signaling delay occurs in session establishment and content delivery. Intelligent prefix caching mechanism expedites immediate channel delivery. Frame transmission delay between content server and user decreases because of availability of proxy server nearer to user premises. P2P network enabled proxy servers exchange requested channel with each other minimizing uncertain congestion and delay that occurs in current delivery architecture. More detailed analysis and implementation remains for further study.

\section{REFERENCES}

[1] T. Wauters, W. Van de Meerssche, F. De Turck, Bart Dhoedt, P. Demeester, T. Van Caenegem, E. Six, "Co-operative Proxy Caching Algorithms for Time-Shifted IPTV Services," EUROMICRO Conference, pp. 379-386, 32nd EUROMICRO Conference on Software Engineering and Advanced Applications, 2006

[2] Zhu Liu; Bin Wei; Yu, H., "IPTV, Towards Seamless Infotainment," Consumer Communications and Networking Conference, 2009. CCNC 2009. 6th IEEE , vol., no., pp.1-5, 10-13 Jan. 2009.

[3] Sentinelli, A.; Marfia, G.; Gerla, M.; Kleinrock, L.; Tewari, S.,"Will IPTV ride the peer-to-peer stream? [Peer-to-Peer Multimedia Streaming]," Communications Magazine, IEEE , vol.45, no.6, pp.86-92, Jun 2007.

[4] Zhi-Li Zhang; Yuewei Wang; Du, D.H.C.; Dongli Su, "Video staging: a proxy-server-based approach to end-to-end video delivery over widearea networks," Networking, IEEE/ACM Transactions on , vol.8, no.4, pp.429-442, Aug 2000.

[5] Song, J. Segment-based proxy caching for distributed cooperative media content servers. SIGOPS Oper. Syst. Rev. 39, 1 (Jan. 2005), 2233. DOI= http://doi.acm.org/10.1145/1044552.1044554.

[6] Tariq, M.A.; Kampmann, M., "A Dynamic Configurable Multimedia Processing Proxy for IPTV Services in Ambient Networks," Mobile and Wireless Communications Summit, 2007. 16th IST , vol., no., pp.1-5, 15 July 2007.

[7] Fastmesh project the Hongkong university of science and technology http://mwnet.cse.ust.hk/Fastmesh/index.html.

[8] Korean patent: User terminal and proxy server of internet protocol television (IPTV) system, and operating method.

[9] Sen, S.; Rexford, J.; Towsley, D., "Proxy prefix caching for multimedia streams," INFOCOM '99. Eighteenth Annual Joint Conference of the IEEE Computer and Communications Societies. Proceedings. IEEE , vol.3, no., pp.1310-1319 vol.3, 21-25 Mar 1999.

[10] B. Wang, S. Sen, M. Adler, and D. Towsley, "Optimal proxy Cache allocation for efficient streaming media distribution," in Proc. IEEE INFOCOM'02, Jun 2002.

[11] H. Uzunalioglu, "Channel Change Delay in IPTV Systems," Consumer Communications and Networking Conference, 2009. CCNC 2009. 6th IEEE ,vol., no., pp.1-6, 10-13 Jan. 2009. 